\title{
Evaluation of a coproantigen enzyme-linked immunosorbent assay for the diagnosis of canine echinococcosis in Iran
}

\author{
M. R. SIAVASHI ${ }^{1}$, GH. R MOTAMEDI ${ }^{2}$ \\ ${ }^{1}$ Department of Parasitology, Pasteur Institute of Iran, Tehran, Iran, E-mail: m_siavashi@hotmail.com; \\ ${ }^{2}$ Department of Parasitology, Razi Vaccine and Serum Research Institute of Iran, Karaj, Iran
}

\begin{abstract}
Summary
Echinococcosis is one of the most important zoonosis in Iran. Due to this fact that providing a reliable diagnostic method for detection of this infection in definitive host is a critical prerequirement for the establishment of appropriate control programs in our country, one hundred and sixteen carnivores including 80 dogs, 27 jackals, 8 foxes and one wolf were collected from rural areas of Hamadan, Azarbaijan and Tehran provinces and examined for Echinococcus granulosus infection. Canine echinococcosis was diagnosed upon direct microscopic examination of intestinal contents and mucosal scraping for adult tapeworms, and a coproantigen detection enzyme linked immunosorbent assay (CA-ELISA) for Echinococcus granulosus. The overall prevalence of canine echinococcosis using the ELISA test was $43.1 \%(50 / 116)$. The relative frequency of canine echinococcosis was $37 \%(43 / 116)$ by microscopic examination.

The sensitivity and specificity of the CA- ELISA test as referenced by necropsy findings was $72.1 \%$ and $74 \%$ respectively. We found this assay to be a very suitable and advantageous method for the surveillance of canine population especially in regions with endemic echinococcosis.
\end{abstract}

Key words: Echinococcus granulosus; canine; coproantigen ELISA; diagnosis; Iran

\section{Introduction}

Cystic echinococcosis has been recognized as a major public health problem in sheep-rising regions of the world (Eckert et al., 2001). Different studies in Iran have shown that echinococcosis is a main health problem among human and domestic livestock in several provinces of our country (Dalimi \& Mobedi, 1992; Dalimi et al., 2002). The development of sensitive and specific diagnostic techniques for detection of echinococcosis in humans, dogs and livestock is important for carrying out field studies and control programs. Diagnosis of human cystic echinococcosis can be made by valid imaging techniques, complemented by relatively sensitive and specific serological assays (Parija et al., 1998; Moro et al., 1999; Eckert et al., 2001). In contrast, diagnosis of echinococcosis in canines has had major limitations. Ante-mortem diagnosis of canine echinococcosis relied so far on the use of arecoline salts for purgation followed by microscopic examination of the purge for presence of the adult tapeworms (WHO, 1981). Although the specificity of this test is $100 \%$, it is biohazardous, time consuming, requires trained personnel and has variable sensitivity. Since an advanced coproantigen enzyme immunoassay has been developed for detection of canine echinococcosis (Allen et al., 1992; Deplazes et al., 1992, 1994), we decided to evaluate this test to recommend it as a reliable method for field study in our country.

\section{Materials and Methods}

Under governmental controls of stray dogs and wild canine in some regions of Iran, 116 adult carnivores including 80 dogs, 27 jackals, 8 foxes and one wolf were shot and collected from villages of Hamadan, Azarbaijan and Tehran provinces during summer and autumn of 2003 and transferred to parasitology laboratory of Razi Vaccine and Serum Institute of Iran (Karaj) by using a plastic bag.

\section{Parasitological examination}

After necropsy of each animal which was performed according to FAO/UNEP/WHO Guidelines (1981), the small intestine was removed, then the whole intestine was opened in a dissecting pan to be examined as follows: The mucosa of the intestine was scraped by a scalpel. Mucosal scraping and intestinal contents were examined under a stereomicroscope. Recovered parasites were fixed in alcohol and stained by Carmine staining method. Echinococcus granulosus adult worms were identified according to the gui- 
delines given by Kumaratilake and Thompson, 1982.

\section{Coproantigen ELISA}

5 to 10 grams of fecal samples were collected from the intestine of each animal and mixed at a $1: 4(\mathrm{~V} / \mathrm{V})$ ratio with buffer solution (PBS containing $0.04 \% \mathrm{NaN}_{3}, 0.05 \%$ bovine hemoglobin (Fluka) and $0.3 \%$ of Tween 20, vigorously shaken and stored deep frozen at $-20^{\circ} \mathrm{C}$ for several weeks until examination. A quantity of $5 \mathrm{ml}$ of prepared fecal material was centrifuged for $10 \mathrm{~min}$ at $3000 \times \mathrm{g}$, and a $2 \mathrm{ml}$ of the supernatant was used for further processing. The examination of the fecal samples for Echinococcus granulosus coproantigen was performed according to Deplazes et al. $(1992,1994)$ by using a commercial kit (Chekit $^{\circledR}$ Echinotest, Dr. Bommeli Diagnostics, Switzerland). The test procedure was performed according to the instructions of the manufacturer including the standard samples which is briefly explained as follows: $80 \mu$ of sample diluent was dispensed into each well (96 well microplates was used), $20 \mu \mathrm{l}$ of positive and negative control (PK7097, $\mathrm{Nk}$ ) or samples were added and the plates were mixed gently. Then the plates were covered and incubated for 90 min at room temperature. After washing the plates for 5 times (by washing solution), $100 \mu$ diluted conjugate (diluted 1:200 in washing buffer) was dispensed into each well and mixed gently. The plates were covered and incubated for $90 \mathrm{~min}$ at RT and post washed for 6 times. At the next stage each well was dispensed by $100 \mu \mathrm{l}$ prewarmed chromogen, mixed gently and incubated for 10 to $20 \mathrm{~min}$ at room temperature. The process was stopped with $50 \mu \mathrm{l}$ stopping solution and read with the ELISA reader (ICN BIOMEDICAL, Switzerland) at wavelength of $405 \mathrm{~nm}$.

\section{Results}

In the present study 116 canines, $62(53.5 \%)$ male and 54 $(46.5 \%)$ female were examined by CA- ELISA. The overall prevalence of canine echinococcosis by ELISA was $43.1 \%$ (50/116 cases including 45 dogs, 3 jackals, 1 fox and 1 wolf) (Table 1). There was no significant difference in prevalence between male and female cases $(\mathrm{P}=0.32$ by the $\chi^{2}$-test). The microscopic examination of intestinal

Table 1. Comparison of CA- ELISA with necropsy in 116 canines in Iran

\begin{tabular}{lccc}
\hline \multicolumn{1}{c}{ Test } & necropsy $(+)$ & necropsy $(-)$ & total \\
\hline CA- ELISA (+) & 31 & 19 & 50 \\
CA- ELISA (-) & 12 & 54 & 66 \\
Total & 43 & 73 & 116 \\
\hline
\end{tabular}

contents and mucosal scraping revealed that $37 \%(43 / 116)$ were infected with E. granulosus tapeworms (Table 1). The worms burdens were between 10 and 250 Echinococcus adult worms per infected cases. The specificity and sensitivity of CA-ELISA test was $74 \%$ and $72.1 \%$ respectively. Also negative predictive value (NPV) of the test was determined as high as $81.8 \%$ and positive predictive value (PPV) was $62 \%$ (Table2).

\section{Discussion}

In the present study the coproantigen prevalence in canine carnivores of three provinces of Azarbaijan, Hamadan and Tehran by ELISA was determined to be $43.1 \%$. Although, with respect to the false positive cases, the real prevalence was $37 \%$, CA-ELISA results are reliable enough to indicate the relative degree of transmission risk to human and livestock in these provinces. These rates were comparable to those of previous studies carried out in the same regions by necropsy, which had shown a range of $22 \%$ to $50 \%$ in different kinds of carnivores (Dalimi et al., 1992, 2002). In the present study the sensitivity of CA- ELISA was $72.1 \%$, which was higher than as reported by Zariffard et al., 1999 (68\%). Studies with different types of CA-ELISAs have revealed sensitivities for E. granulosus detection of $88 \%$ in Peru (Lopera et al., 2003), of $83 \%$ in Cyprus (Christofi et al., 2002), of $77 \%$ in Uruguay (Craig et al., 1995), of $63 \%$ in Spain (Deplazes et al., 1994) and of $62 \%$ in Jordan (El-Shehabi et al., 2000). Various studies have shown that the sensitivity of CA - ELISA is strongly dependent on the worm burden. Some of these studies demonstrated a significantly increased sensitivity in dogs with E. granulosus burdens above 100 worms per animal (Christofi et al., 2002; Deplazes et al., 1994). We observed similar results, but the difference between light ( $<50$ worms), moderate $(50-100$ worms $)$ and severe $(>$ 100 worms) burdens was not significant $(P=0.45)$. According to Christofi et al., 2002, areas with low prevalence of canine echinococcosis, exhibit low sensitivity and positive predictive value of CA-ELISA, but specificity and negative predictive values were high. This finding was also coinciding with our study in that the prevalence was as high as $43.1 \%$, and sensitivity and specificity were $72.1 \%$ and $74 \%$, respectively. In our study negative results in CA - ELISA exhibited a predictive value of $(81.8 \%)$. Ideally, field prevalence should be assessed in canine populations by necropsy or in animals purged with arecoline (El-Shehabi et al., 2000). However, these approaches are not well acceptable to the community, so that CA-ELISA will be more suitable especially for endemic regions with higher prevalence of canine echinococcosis.

Table 2. Coproantigen ELISA test validation for detection of E.granulosus coproantigens in fecal sample of canines

\begin{tabular}{cccccc}
\hline Total No & ELISA + & Sensitivity & Specificity & PPV & NPV \\
\hline 116 & $43.1 \%(50 / 116)$ & $72.1 \%(31 / 43)$ & $74 \%(51 / 73)$ & $62 \%(31 / 50)$ & $81.8 \%(54 / 66)$ \\
\hline
\end{tabular}




\section{References}

Allen, J. C., Craig, P. S., Garcia, N., Mencos, F., LiU, D., WANG, Y., Wen, Y., Zhou, P., Stringer, R., Rogan, M., ZEYHLE, E. (1992): Coproantigen detection for immunodiagnosis of echinococcosis and taeniasis in dogs and humans. Parasitology, 104: 347 - 355

Christofi, G., Deplazes, N., Christofi, N., Tanner, I., ECONOMIDES, P., ECKERT, J. (2002): Screening of dogs for Echinococcus granulosus coproantigen in low endemic situation in Cyprus. Vet. Parasitol., 104: 299 - 306

Craig, P. S., Gassar, R. B., Parada, L., Caberera, P., Parietti, S., Borgues, C., Acuttis, A., Agulla, J., Snowden, K., Pallilo, E. (1995): Diagnosis of canine echinococcosis: comparison of coproantigen and serum antiboby tests with arecoline purgation in Uruguay. Vet. Parasitol, 56: 293 - 301

DALIMI, A., MoBedI, I. (1992): Helminth parasites of carnivores in northern Iran. Ann. Med. Parasitol., 86: 395 $-397$

Dalimi, A., Motamedi, Gh., Hosseini, M., MoHammaDian, B., MaleKi, H., Ghamari, Z., Ghaffari Far, F. (2002): Echinococcosis/Hydatidosis in western Iran. Vet. Parasitol., 105: 161 - 171

Deplazes, P., Gottestin, B., Eckert, J., Jenkins, D. J., Ewald, D., Jimenez-PALACios, S. (1992): Echinococcus multilocularis coproantigens detection by enzyme linked immunosorbent assay in fox, dog and cat populations. $J$. Parasitology, 85: 115 - 121

Deplazes, P., Jimenes-Palacios, S., Gottestin, B., SKAGGS, J., ECKERT, J. (1994): Detection of Echinococcus coproantigens in stray dogs of northern Spain. Appl. Parasitol., 35: 297 - 301

Eckert, J., Deplazes, P., Craig, P.S., Gemmell, M. A., Gottstein, B., Health, D., Jenkins, D.J., Kamiya, M., LightowlERs, M. (2001): Echinococcosis in animals: cli- nical aspects, diagnosis and treatment, In ECKERT, J., GEMMEL, M. A., Meslin, F.X., PAWlowski, Z. (Eds), WHO/ OIE Manual on echinococcosis in humans and animals: A public health problem of global concern. World Organization for Animal Health, Paris: 72 - 99

El-Shehabi, F. S., Kamhawi, S. A., Schantz, P. M., Craig, P. S., AbDel-Hafez, S. H. (2000): Diagnosis of canine echinococcosis: comparison of coproantigen detection with necropsy in stray dogs and red foxes from northern Jordan. Parasite, 7: 83 - 90

Kumaratilake, L. M., Thompson, R. C. A. (1982): A review of taxonomy and speciation of the genus Echinococcus rudolphi, 1801. Z. Parasitenkunde, 68: 121 - 146

Lopera, L., Moro, P. L., Chavez, A., Monres, G., GoNZALES, A., GilmAN, R.H. (2003): Field evaluation of a coproantigen enzyme-linked immunosorbent assay for diagnosis of canine echinococcosis in a rural Andean village in Peru. Vet. Parasitol., 117: 37 - 42

Moro, P. L., Bonifacio, N., Gilman, R. H., Lopera, L., Silva, B., Takumoto, R., Verastegui, M., CABrera, L. (1999): Field diagnosis of Echinococcus granulosus infection among intermediate and definitive hosts in a endemic focus of human cystic echinococcosis. Trans. R. Soc. Trop. Med. Hyg., 93: $611-615$

PARIJA, S. C. (1998): A review of some simple immunoassays in the serodiagnosis of cystic hydatid disease. Acta Tropica, 7: $17-24$

WHO (1981) In ECKerT, J., Gemmell, M. A., Soulsby, E. J. L. (Eds), Guidelines for surveillance, prevention and control of echinococcosis/Hydatidosis. FAO/UNEP/WHO, Geneva, Switzerland, 265

ZarifFARD, M. R., DePlazes, P., BASHER, N., MotAMEDI, G.R., NAZARI, J.H. (1999): Detection of Echinococcus granulosus in carnivores in Iran, Arch. Int. Hydatidol., 23: 264 